\title{
L'emploi des personnes handicapées dans la fonction publique
}

Entre quota et non-discrimination, quelles pratiques des employeurs ?

Quota and Non-Discrimination Approach on Employment of Persons with

Disabilities in the French Public Service. What Are the Employers' Practices?

\section{Fanny Jaffrès et Marie-Renée Guével}

\section{OpenEdition}

Journals

Édition électronique

URL : http://journals.openedition.org/travailemploi/7759

DOI : 10.4000/travailemploi.7759

ISSN : 1775-416X

Éditeur

DARES - Ministère du Travail

Édition imprimée

Date de publication : 1 octobre 2017

Pagination : 33-57

ISSN : 0224-4365

\section{Référence électronique}

Fanny Jaffrès et Marie-Renée Guével, «L'emploi des personnes handicapées dans la fonction publique », Travail et Emploi [En ligne], 152 | octobre-décembre 2017, mis en ligne le 11 juillet 2019, consulté le 05 septembre 2019. URL : http://journals.openedition.org/travailemploi/7759 ; DOI : 10.4000/ travailemploi.7759 


\title{
L'emploi des personnes handicapées dans la fonction publique
}

\author{
Entre quota et non-discrimination, \\ quelles pratiques des employeurs?
}

\author{
Fanny Jaffrès", Marie-Renée Guével ${ }^{* *}$
}

\begin{abstract}
Basé sur une enquête de terrain conduite auprès d'employeurs publics, cet article étudie comment ils se saisissent de leurs obligations en matière d'emploi des personnes handicapées. La politique d'emploi des personnes handicapées est en effet traversée en France par une tension entre une logique catégorielle de quota et une logique universaliste de non-discrimination portée par la notion d' « aménagement raisonnable » des emplois. La première logique est bien connue et les obligations qui l'accompagnent sont de mieux en mieux respectées par les employeurs de la fonction publique, comme en témoigne la hausse continue du taux d'emploi de travailleurs handicapés qui y est observée. La deuxième logique est émergente, mais plusieurs indices laissent penser qu'elle pourrait être particulièrement développée dans la fonction publique, qui proposerait plus souvent un environnement de travail adapté à chacun, quel que soit son handicap. Partant de ces hypothèses, nous avons conduit une campagne d'entretiens dans sept établissements publics auprès des acteurs concernés par la politique d'emploi des personnes handicapées. Ces entretiens ont été complétés par un recueil de documents (conventions, plaquettes de communication, etc.) et d'observations. Le matériau fait ressortir une situation complexe, qui contraste avec l'évolution largement favorable de la proportion de travailleurs handicapés. Il apparaît notamment que si les quotas se révèlent être des instruments puissants permettant d'engager un changement, la notion d'aménagement raisonnable et l'approche en termes de non-discrimination peinent à s'implanter. L'analyse des facteurs qui influencent les stratégies des employeurs met en avant l'importance des enjeux économiques et financiers et vient questionner la compatibilité de l'organisation de la fonction publique et de son mode de gestion des ressources humaines avec la construction d'une approche ambitieuse de non-discrimination et la prise en compte des besoins particuliers des personnes handicapées.
\end{abstract}

\footnotetext{
* Université de Rennes, École des hautes études en santé publique (EHESP), CNRS, Arènes UMR 6051; Fanny.Jaffres@ehesp.fr.

** Université de Rennes, École des hautes études en santé publique (EHESP), CNRS, Arènes UMR 6051 ; Marie-Renee.Guevel@ehesp.fr.
} 
D 'abord conçu comme un enjeu de solidarité nationale, le handicap s'est, depuis la fin du XX $\mathrm{X}^{\mathrm{e}}$ siècle, inscrit dans une logique de politique d'emploi. Engagées dans un mouvement d'activation des dépenses sociales, les politiques publiques des pays européens ont aujourd'hui pour objectif de promouvoir l'intégration sociale, économique et professionnelle des personnes handicapées (RowELL, 2016). Pour soutenir cet objectif, la France a fait le choix, depuis 1924, d'une obligation d'emploi basée sur un quota, et assortie de sanctions financières en cas de non-respect et d'aides soutenant les actions des employeurs. Cette logique s'applique à la fonction publique seulement depuis la loi du 11 février 2005 pour l'égalité des droits et des chances, la citoyenneté et la participation des personnes handicapées, qui est à l'origine du Fonds pour l'insertion des personnes handicapées dans la fonction publique (FIPHFP), chargé de collecter les contributions et de soutenir financièrement les actions des employeurs publics. Cette même loi, en application de la directive européenne 2000/78/CE en faveur de l'égalité de traitement en matière d'emploi et de travail, a introduit une obligation d' « aménagement raisonnable » de l'environnement de travail (voir infra) sur la base du principe de non-discrimination. Il est en effet aujourd'hui attendu des employeurs qu'ils prennent des mesures appropriées pour garantir l'égalité de traitement entre les salariés quel que soit leur état de santé ou leur handicap.

Le rapport des employeurs à ces obligations en matière d'emploi des personnes handicapées est complexe. L'évolution du taux d'emploi de bénéficiaires de l'obligation d'emploi (BOE), notamment au sein de la fonction publique, suggère qu'ils sont enclins à la respecter. En effet, la part de ces bénéficiaires dans l'effectif salarié global est en augmentation. Alors qu'entre 2010 et 2013 dans le secteur privé, le taux d'emploi des BOE est passé de 2,9\% à 3,3\%, il est passé, pour les trois fonctions publiques réunies, de 4,2 \% à 4,9 \% (BARHOumi, CHABAnOn, 2015). Pourtant, l'existence d'attitudes discriminatoires de la part de certains employeurs à l'égard des travailleurs handicapés, démontrées il y a plus d'une vingtaine d'années (RAVAUD, MADiot, ViLle, 1992), perdure. Dans son rapport, d'activité de 2016, le Défenseur des droits (2017) - qui a pour missions de défendre les personnes dont les droits ne sont pas respectés et de favoriser l'égalité de tous dans l'accès aux droits - montre par exemple que le handicap et l'état de santé constituent, après l'origine, les principaux motifs de saisine pour discrimination dans l'emploi.

Les recherches déjà menées sur l'emploi des personnes handicapées en France concernent principalement le secteur privé. Elles font état d'une situation préoccupante et mettent notamment en lumière les difficultés rencontrées par des travailleurs handicapés. Les travaux de Claudia BARril et Jean-Luc METZGER (2004) mettent en évidence la complexité de l'insertion des travailleurs non-voyants et sourds dans des structures et des organisations du travail qui évoluent en permanence, notamment en ce qui concerne les outils informatiques. Ces auteurs soulignent que la perpétuation des compétences des travailleurs non-voyants et sourds nécessite la mise en place d'un collectif d'apprentissage. Or, plusieurs travaux, dont ceux de Sophie DALLE-NAzÉBI (2013) sur les travailleurs sourds, indiquent qu'un tel collectif se constitue souvent 
difficilement, en raison d'une incompréhension de la part des salariés non-handicapés quant aux compétences et aux besoins des salariés sourds. L'auteure explique comment l'asymétrie entre sourds et non-sourds conduit à des situations de «violence ordinaire », faites d'absence de communication, d'évitement et de compassion. Comme le montrent Sylvain KeRBOURC'H et S. DALLE-NAZÉBI (2014), l'intégration des personnes sourdes est essentiellement permise par un « travail en plus » de leur part, bien souvent invisible du fait d'une définition du handicap essentiellement centrée sur l'individu. Bien qu'il existe une contrainte légale à agir pour l'emploi des personnes handicapées, cette question est largement perçue comme un enjeu individuel et fait rarement l'objet d'un traitement au niveau organisationnel.

De nombreux travaux soulignent la variété des attitudes des employeurs face aux objectifs de diversité (Ewick, Silbey, 1998 ; Point, Charles-Fontaine, BerthÉlÈme, 2010 ; Barel, Frémeaux, 2013). Par exemple, Yvan Barel et Sandrine FrémeauX (2013) proposent, à propos du management de la diversité dans les entreprises, une typologie composée de quatre catégories d'employeurs : les proactifs, qui mènent une véritable politique d'intégration ; les porteurs d'une attitude réactive contrainte, qui souffrent d'un déficit managérial ; les porteurs d'une attitude réactive instrumentale, qui effectuent des recrutements ciblés ; enfin, les porteurs d'une attitude réactive passive, qui ne mènent aucune action. Dans la catégorie de ceux présentant une attitude proactive, Y. BAREL et S. FrÉMEAUX ne placent qu'une minorité d'employeurs. Ces résultats, qui concernent le secteur privé, rejoignent ceux obtenus par Annie CORNET et Manal El Aвbоubi (2012) dans leur étude des pratiques de gestion de la diversité dans les secteurs publics belge, français et canadien. Ces auteures montrent que si certains employeurs publics mettent en place des actions de fond visant à changer les structures et les procédures (diversification des canaux de recrutement, formation à la diversité des responsables du recrutement, révision des procédures d'évolution de carrière, etc.), les actions les plus fréquemment mises en place sont les moins contraignantes, celles qui visent simplement des changements individuels et notamment la montée en compétences des personnes handicapées. Les politiques d'emploi des travailleurs handicapés restent isolées et ne sont souvent pas considérées dans les évaluations de performance des entreprises (MORIN, 2006). De manière générale, les employeurs semblent continuer à penser en termes d'égalité des places et non en termes d'égalité des chances (CAILLEBA, CuEvas, 2009), c'est-à-dire qu'ils tentent d'atteindre le quota sans chercher à permettre une pleine inclusion des personnes handicapées dans le collectif de travail. Par rapport à ces travaux brièvement présentés ici et qui portent majoritairement sur le secteur privé, la spécificité de cet article est de porter sur la fonction publique. Celle-ci représente, en effet, une part non négligeable de l'emploi en France (20 \%, DGAFP, 2015) et constitue un cadre d'emploi spécifique par rapport au secteur privé, du fait de son devoir d'égalité des chances (POUGET, 2005). Elle est régie par des règles différentes du Code du travail : les statuts de la fonction publique. Julien POUGET (2005) souligne ainsi, entre autres spécificités, que le recrutement dans la fonction publique se fait majoritairement sur concours, que 
les avancements de carrières sont régis par des grilles indiciaires, que la fonction publique emploie davantage de cadres que le secteur privé et qu' elle est soumise à une obligation de reclassement. En matière de santé au travail, les travaux concernant les effets d'un événement de santé sur la trajectoire professionnelle, et notamment une étude quantitative récente de l'effet d'un handicap sur l'emploi (BARNAY, DUGUET et $a l ., 2015)$, montrent que si les personnes en emploi dans le secteur privé voient leur situation professionnelle se dégrader dans les cinq années suivant la survenue d'un handicap, celles employées dans le secteur public se maintiennent en emploi suite à ce même événement.

Ces spécificités et la forte évolution des taux d'emploi de BOE dans la fonction publique amènent à questionner la manière dont les employeurs publics se saisissent de leurs obligations en matière d'emploi des personnes handicapées et les facteurs qui influencent leurs pratiques. La principale hypothèse défendue dans cet article est que les chiffres positifs de l'action en faveur de l'emploi des personnes handicapées recouvrent une réalité contrastée ${ }^{1}$. Cet article s'appuie sur une étude conduite en 2015 selon une méthodologie d'enquête qualitative (encadré 1 ), auprès de sept employeurs publics (tableau 1) issus des trois fonctions publiques et présentant des missions (administration locale, politique nationale, culture, enseignement, soin), des tailles (de 200 à plus de 20000 agents) et des taux d'emploi de BOE variés.

Après une présentation du contexte réglementaire et politique français en matière de handicap et d'emploi des personnes handicapées, l'article montre la mise en œuvre progressive d'une logique de non-discrimination allant au-delà de l'approche catégorielle par quota au sein des stratégies des employeurs publics enquêtés et propose une explication de la variété de ces stratégies, avant d'ouvrir en conclusion sur l'actuel - et nécessaire - tournant des politiques publiques du handicap ${ }^{2}$.

\section{ENCADRÉ}

\section{Méthodologie d'enquête}

Notre étude a été conduite auprès de sept employeurs publics selon une méthodologie d'enquête qualitative : deux collectivités territoriales, deux centres hospitaliers et trois employeurs de la fonction publique d'État (un ministère, un établissement d'enseignement supérieur et de recherche et un établissement culturel). Au sein de chaque structure, des entretiens semi-directifs individuels (parfois avec deux personnes) ont été conduits de décembre 2014 à décembre 2015. Ils ont duré en moyenne 50 minutes et ont permis d'interroger différentes catégories d'acteurs. D'une part, nous nous sommes entretenues avec

1. Cette étude a été menée dans le cadre du programme de recherche sur l'emploi des personnes handicapées financé par le FIPHFP et conduit par l'EHESP.

2. Nous souhaitons ici remercier les rapporteurs pour leurs retours et leurs suggestions de lecture qui ont grandement contribué à la clarification de notre argumentation. 
les personnes qui décident et mettent en œuvre la stratégie de l'établissement en matière d'emploi des personnes handicapées, c'est-à-dire les référents handicap, les directeurs et directrices des ressources humaines (DRH), les médecins du travail, les psychologues du travail, les ingénieurs hygiène et sécurité ainsi que toute autre personne qui pouvait nous être recommandée. Le but de ces entretiens était de cerner les enjeux en matière d'emploi pour l'organisation dans son ensemble. D'autre part, nous avons interrogé des travailleurs handicapés et leur encadrement afin de croiser les regards sur les situations et de mieux appréhender l'application concrète des stratégies des employeurs. Une quinzaine d'entretiens ont ainsi été menés au sein de chaque structure et une à sept situations concrètes y ont été étudiées en fonction de la taille de l'employeur. De plus, nous avons pris en note des données d'observation et collecté des documents produits par les établissements pouvant éclairer les questions liées à l'emploi des personnes handicapées, notamment les conventions FIPHFP et les plaquettes de communication.

TABLEAU 1 - Répartition du matériau recueilli

\begin{tabular}{|c|c|c|c|c|c|}
\hline & $\begin{array}{l}\text { Fonction } \\
\text { publique }\end{array}$ & $\begin{array}{l}\text { Nombre } \\
\text { d'agents }\end{array}$ & $\begin{array}{l}\text { Taux d'emploi } \\
\text { légal de } \\
\text { travailleurs } \\
\text { handicapés }\end{array}$ & $\begin{array}{l}\text { Nombre de } \\
\text { personnes } \\
\text { rencontrées }\end{array}$ & $\begin{array}{c}\text { Nombre de } \\
\text { situations } \\
\text { de handicap } \\
\text { étudiées }\end{array}$ \\
\hline Ministère $\mathrm{Y}^{*}$ & État & $\begin{array}{l}\text { Plus de } 20000 \\
\text { sur plusieurs } \\
\text { sites répartis sur } \\
\text { la France entière }\end{array}$ & $2,5 \%$ & 18 & 7 \\
\hline $\begin{array}{l}\text { Établissement public } \\
\text { culturel Chêne }\end{array}$ & État & $\begin{array}{l}\text { Entre } 250 \text { et } \\
1000 \text { sur un seul } \\
\text { site }\end{array}$ & $\begin{array}{l}\text { Moins } \\
\text { de } 3 \%\end{array}$ & 17 & 6 \\
\hline $\begin{array}{l}\text { Établissement } \\
\text { d'enseignement supérieur } \\
\text { et de recherche Confucius }\end{array}$ & État & $\begin{array}{l}\text { Entre } 250 \text { et } \\
1000 \text { sur } \\
\text { plusieurs sites } \\
\text { éloignés }\end{array}$ & $4 \%$ & 14 & 5 \\
\hline Centre hospitalier Beta ${ }^{*}$ & Hospitalière & $\begin{array}{l}\text { Entre } 1000 \text { et } \\
5000 \text { sur un seul } \\
\text { site }\end{array}$ & $4,9 \%$ & 20 & 4 \\
\hline Centre hospitalier Alpha ${ }^{*}$ & Hospitalière & $\begin{array}{l}\text { Entre } 1000 \text { et } \\
5000 \text { sur } \\
\text { plusieurs sites } \\
\text { proches }\end{array}$ & $5,4 \%$ & 13 & 5 \\
\hline Collectivité territoriale $2^{*}$ & Territoriale & $\begin{array}{l}\text { Moins de } 250 \\
\text { sur plusieurs } \\
\text { sites proches }\end{array}$ & $5,8 \%$ & 6 & 2 \\
\hline Collectivité territoriale $1^{*}$ & Territoriale & $\begin{array}{l}\text { Entre } 1000 \text { et } \\
5000 \text { sur } \\
\text { plusieurs sites } \\
\text { proches }\end{array}$ & 6 à $9 \%$ & 9 & 3 \\
\hline Total & & & & 96 & 32 \\
\hline
\end{tabular}

"L'ensemble des établissements ont été anonymisés.

Source : données fournies par les établissements en 2015 ; enquête des auteures. 


\section{La complexité du contexte réglementaire et politique en matière d'emploi des personnes handicapées}

\section{Des tensions entre les dispositifs}

L'action publique à destination des personnes handicapées s'inscrit dans un contexte législatif et réglementaire complexe. Comme le met en avant Pierre-Yves BAUDOT (2016), la politique du handicap s'est établie comme une « juxtaposition d'actions publiques éparses ». Les dispositifs de ce champ visent des populations différentes, puisque certains ont initialement été créés pour les accidentés du travail, d'autres pour les invalides et mutilés de guerre, d'autres encore pour les invalides civils. Même s'ils ont été peu à peu élargis pour permettre une prise en charge équivalente de ces différentes populations, jusqu'à parfois se recouper, un flou persiste autour du public cible. De plus, bien que la loi de 2005 ait tenté d'instaurer une logique de service où les besoins des personnes sont au cœur des pratiques, «l'emprise administrative sur la définition de la population ciblée » reste forte (BAUDOT, 2016). Ce processus est d'autant plus complexe qu'il fait intervenir plusieurs administrations compétentes pour déterminer cette catégorie, qui travaillent de façon indépendante (maisons départementales des personnes handicapées et Assurance maladie notamment).

La complexité de ce champ tient aussi au fait que les dispositifs n'ont pas été construits au même moment et reposent sur des paradigmes différents. Comme le montrent Myriam Winance, Isabelle Ville et Jean-François RaVaud (2007), les politiques conduites en France ont dans un premier temps été catégorielles, c'està-dire construites sur un modèle médical du handicap, donc centrées sur l'individu et ses déficiences et où la reconnaissance médicale prévalait. Puis, inspirées par les questions soulevées dans les pays anglo-saxons, elles ont pris un tournant universaliste en s'appuyant sur un modèle social du handicap qui le définissait comme le résultat d'un environnement inadapté. Enfin, les politiques ont glissé vers un traitement plus personnalisé, fondé sur le concept de « situation de handicap » qui met sur le même plan les facteurs individuels et environnementaux. Loin de se substituer les unes aux autres, ces politiques se sont accumulées pour constituer un ensemble complexe où les dispositifs peuvent aussi bien se montrer complémentaires que divergents.

L'exemple des dispositifs pour l'emploi des personnes handicapées reflète cette diversité de logiques. Dans ce domaine, depuis la fin du $\mathrm{xx}^{\mathrm{e}}$ siècle, la logique de solidarité s'est peu à peu effacée devant un objectif d'intégration : les politiques publiques doivent permettre voire inciter les personnes handicapées à mener une vie ordinaire grâce au travail. Pour créer des conditions favorables à l'emploi de ces personnes, elles se sont dans un premier temps basées sur le principe de réadaptation des corps puis, après les Trente Glorieuses, le principe de non-discrimination a pris de plus en plus d'importance (VILle, Fillion, RaVAud, 2014). Là encore, les dispositifs se sont juxtaposés et, avec eux, les modèles du handicap, médical et social, parfois opposés, se sont superposés. L'articulation de deux instruments clés de l'action publique en 
matière d'emploi des personnes handicapées - le quota de travailleurs et la notion d'aménagement raisonnable - illustre cette tension.

\section{Le quota : un instrument puissant mais contestable}

Depuis très longtemps, la politique d'emploi des personnes handicapées repose sur une obligation d'emploi associée à un quota, mais la population visée par un tel dispositif a constamment évolué. Le quota fixé pour la première fois par la loi du 26 avril 1924, qui concernait les administrations et les entreprises de plus de dix salariés, était de $10 \%$ et visait les mutilés de guerre et les accidentés du travail. Au fil des années, le dispositif a été renforcé. Certes, la loi du 10 juillet 1987 abaisse le quota à $6 \%$ et réduit son champ d'application aux établissements de plus de vingt salariés. Mais dans le même temps, elle instaure une contribution financière pour les employeurs privés ne le respectant pas, ainsi que des aides pour la mise en place d'actions favorisant l'emploi des personnes handicapées. La gestion de ces fonds est confiée à l'Association de gestion du fonds pour l'insertion des personnes handicapées (Agefiph). La loi de 2005 va également dans le sens d'un renforcement de ce dispositif : elle révise le calcul du montant de la contribution de manière à l'alourdir et applique la logique de sanctions et d'aides financières à la fonction publique en créant le FIPHFP.

La contribution en cas de non-respect du taux de $6 \%$ de BOE est dite « volontaire ». Selon les textes législatifs, elle est une réponse à l'obligation d'emploi au même titre que l'emploi direct de travailleurs handicapés, la sous-traitance avec le secteur protégé ou adapté et certaines dépenses liées à l'insertion de personnes handicapées ou à l'aménagement de postes pour permettre leur maintien dans l'emploi. Cependant, sauf exemption particulière, en cas de non-respect du quota, les employeurs sont dans l'obligation de la verser $^{3}$. Elle est donc vécue comme une pénalité financière et s'avère incitative dans la mesure où elle conduit les employeurs à engager des actions :

« On voit que [dans] la déclaration en 2011, notre contribution était à peu près de 400000 euros [...] on avait une contribution qui est énorme, en tout cas, pour l'hôpital Beta, ça faisait presque dix postes d'aides-soignants ! »

(Eulalie, référente handicap de la fonction publique hospitalière, septembre 2015)

« Le point de départ, le point de départ de tout ça, c'était... c'est tout bête, c'était une réflexion sur les sommes considérables qu'on payait à l'époque et qu'on allait

3. Calculée en fonction du nombre de BOE manquants, cette contribution annuelle peut représenter un montant important pour les employeurs : 400 fois le Smic horaire par BOE manquant pour les entreprises de 20 à 199 salariés ; 500 fois le Smic horaire par BOE manquant pour les entreprises de 200 à 749 salariés ; 600 fois le Smic horaire par BOE manquant pour les entreprises de plus de 750 salariés. Si pour les entreprises comptant plus de $80 \%$ d'emplois exigeant des conditions d'aptitude particulières, la contribution est plus faible (40 fois le Smic horaire par BOE manquant), l'inaction prolongée est sévèrement sanctionnée. Quels que soient leur taille ou type de main-d'œuvre, les employeurs qui ne réalisent aucune action sur trois années consécutives doivent payer une contribution de 1500 fois le Smic horaire par unité manquante. 
continuer à payer et qui seraient de plus en plus importantes en termes de non-respect de l'effectif des $6 \%$.»

(Patrick, ex-DRH de la fonction publique hospitalière, avril 2015)

Outre la collecte de cette contribution, le FIPHFP soutient financièrement les employeurs qui mettent en œuvre des politiques d'inclusion professionnelle des personnes handicapées. Des aides peuvent être demandées de manière ponctuelle après la mise en place d'actions, ou alors versées en amont des actions grâce à une convention triennale entre l'établissement et le FIPHFP ${ }^{4}$. Leur montant est un enjeu clé pour les personnes en charge de ces actions. Le montant des aides est en effet un attribut de pouvoir qui permet l'action. Le référent handicap de l'établissement culturel nous expliquait ainsi que dire qu'il gérait des centaines de milliers d'euros en tant que responsable de la convention lui donnait du crédit auprès de ses interlocuteurs, en interne comme en externe.

Grâce à ce système de sanctions et d'incitations financières, le quota apparaît comme un instrument efficace, dans le sens où il a imposé au fil du temps « des règles, des normes et des procédures, des séquences d'action standardisées, plus ou moins coordonnées et contraignantes, qui gouvernent des interactions entre individus » (LASCOUMES, Le Galès, 2010, p. 98). Il convient toutefois de s'interroger sur la population qui en bénéficie. Depuis 1924, le champ des bénéficiaires de l'obligation d'emploi (BOE) s'est successivement élargi : aux victimes civiles de la Seconde Guerre mondiale en 1948, aux veuves et orphelins de guerre en 1955, aux handicapés physiques et mentaux en 1957, aux personnes atteintes de maladies professionnelles en 1975 et enfin aux victimes civiles d'attentats terroristes en 1987 (CALVÈs, 2016). Cette extension lui a peu à peu fait perdre en unité. Le vocable est d'ailleurs significatif : le terme de «bénéficiaire de l'obligation d'emploi » a été préféré à celui de « travailleur handicapé » et, dans la définition de ce terme, c'est la reconnaissance administrative qui prévaut. Or, celle-ci ne suffit pas à définir le handicap, puisque les personnes déclarant une déficience, celles déclarant une incapacité ou encore celles bénéficiant d'une reconnaissance de leur handicap constituent des populations qui ne se recouvrent pas pleinement (VIlle, RaVAud, Letourmy, 2003). De plus, la reconnaissance administrative du handicap n'implique pas forcément sa reconnaissance par l'environnement de travail (GARDIEN, 2006), ni par les personnes elles-mêmes, pour qui le statut administratif peut n'être qu'un moyen parmi d'autres d'obtenir de l'aide, voire de conserver un emploi (BERTRAND, CARADEC, EIDELIMANn, 2014). De fait, les bénéficiaires de l'obligation d'emploi rencontrés dans le cadre de notre enquête présentent des incapacités et des besoins d'aménagement variés, ainsi qu' un handicap plus ou moins reconnu par l'entourage ; des personnes présentant une cécité ou une surdité profonde aux agents recevant une rente pour accident du travail mais ne présentant, au moment de l'enquête, aucune limitation dans l'exercice de leurs fonctions.

4. La possibilité de conventionnement est soumise, notamment, à la taille de l'employeur. 
Par ailleurs, ce dispositif peut être perçu comme porteur d'une conception incompatible avec une logique de droits. Selon Katharina HeYer, le quota, basé sur une logique de traitement spécifique, serait associé à un modèle de protection sociale opposé à un modèle des droits. Il serait ainsi incompatible avec une approche en termes d'intégration et de non-discrimination (traduction commentée de BAUDOT et al., 2013). On peut en effet s'interroger sur la capacité des BOE à être perçus et à se percevoir euxmêmes comme des agents à part entière alors qu'ils reçoivent un traitement spécifique. P.-Y. Baudot, Céline Borelle et Anne Revillard (2013) montrent toutefois que modèle des droits et modèle de protection sociale ne sont pas radicalement opposés et que d'autres constructions des droits, hybrides, sont possibles, comme celle des droits sociaux. Nous faisons le même constat : en pratique, les deux modèles cohabitent, étant chacun symbolisés par deux instruments emblématiques - le quota, comme nous venons de l'exposer, et la notion d'aménagement raisonnable.

\section{La notion d'aménagement raisonnable ou l'introduction d'une logique de non-discrimination}

En parallèle du renforcement de l'obligation d'emploi et de la logique de quota, la loi de 2005 a introduit la notion d'aménagement raisonnable, transposant ainsi en droit français les principes de non-discrimination inscrits dans la directive européenne 2000/78/CE en faveur de l'égalité de traitement en matière d'emploi et de travail. Ce faisant, la loi a opéré un changement de paradigme dans l'appréhension du handicap, avec le passage d'un modèle individuel à un modèle interactionnel, si ce n'est social. Le handicap n'est plus considéré comme un enjeu uniquement lié à l'individu et à sa ou ses déficience(s), mais comme une situation liée tant aux caractéristiques de la personne qu'à celles de son environnement. La loi définit en effet le handicap comme une « limitation d'activité ou [une] restriction de participation à la vie en société subie dans son environnement par une personne en raison d'une altération substantielle, durable ou définitive d'une ou plusieurs fonctions physiques, sensorielles, mentales, cognitives ou psychiques, d'un polyhandicap ou d'un trouble de santé invalidant ». De plus, la loi de 2005 rappelle le droit des personnes handicapées à accéder sans discrimination à l'emploi. En vertu de cette définition situationnelle du handicap, il est donc attendu des employeurs qu'ils prennent des mesures pour garantir une égalité de traitement des travailleurs en situation de handicap. L'article 24 de la loi prévoit en effet des aménagements des emplois ou des postes, qui sont désignés sous le vocable de «mesures appropriées » :

«Afin de garantir le respect du principe d'égalité de traitement à l'égard des travailleurs handicapés [...], les employeurs prennent, en fonction des besoins dans une situation concrète, les mesures appropriées pour permettre aux travailleurs $[\ldots]$ d'accéder à un emploi ou de conserver un emploi correspondant à leur qualification, de l'exercer ou d'y progresser ou pour qu'une formation adaptée à leurs besoins leur soit dispensée, sous réserve que les charges consécutives à la mise en œuvre 
de ces mesures ne soient pas disproportionnées, compte tenu des aides qui peuvent compenser en tout ou partie les dépenses supportées à ce titre par l'employeur. »

La portée de cet instrument s'avère relativement faible en comparaison de celle du quota, comme nous le verrons. Son caractère modéré est une première explication. Les employeurs restent libres du choix des aménagements mis en œuvre, qui ne doivent pas entraîner de charges « disproportionnées ». Cette faible portée s'explique également par une méconnaissance de la part des employeurs. Les travaux de Delphine THIVET et Emmanuelle Fillion (2017) sur le secteur public sanitaire et médico-social l'ont déjà montré et nos résultats le confirment pour les trois versants de la fonction publique. Beaucoup d'agents témoignent de la difficulté à obtenir un aménagement :

«Les recommandations de la médecin ne sont pas assez claires : on est déclaré apte au poste... Aucune préconisation, ni sur les horaires, ni sur du port de charge, ni sur des aménagements, rien... apte au poste. Donc, si on veut s'y prendre au sens de la lettre, on m'envoie en Europe, on me fait faire tout ce qu'on veut... Alors les réunions si, ça, c'est quelque chose : personne ne comprend que passer deux heures, ou trois, sur une chaise comme vous êtes assise, pour moi, c'est insupportable. »

(Cheffe de projet de la fonction publique d'État, juillet 2015) «- Ici, vous avez un aménagement spécifique [...] ?

- Ouais, qui a été très, très, très, très, très, très dur à obtenir (en riant). Je ne sais pas si j'ai dit assez de "très" d'ailleurs. Je suis arrivée déjà euh... ben, clairement le handicap fait peur. Donc, ma directrice était - ça reste secret, hein, ce qu'on se dit ? - elle a tout fait pour me décourager mais vraiment, je n'ai jamais été traitée comme j'ai été traitée, quoi. [...] j'ai été extrêmement mal accueillie. »

(Enseignante de la fonction publique d'État, novembre 2015)

« Il y a des fois des aménagements qui sont préconisés dans les fiches d'aptitude mais la faisabilité est difficile à faire, pas par manque de moyens, mais... Et ça peut être très pragmatique : il y a une difficulté à mettre en œuvre, par exemple, un aménagement de poste, mais en même temps, je dirais que ce n'est pas du ressort du médecin du travail. Il n'a pas à se plier par rapport à des difficultés de mise en place du poste aménagé [...]. Souvent, dans les entreprises privées ou les hôpitaux, on dit : "Oui, mais on ne peut pas le mettre en place." On reprocherait même au médecin d'avoir écrit ce qu'il a écrit parce qu'on dit : "Ce n'est pas possible votre truc, on ne va pas y arriver." »

(Médecin du travail de la fonction publique d'État, juillet 2015).

La difficile application de l'obligation d'aménagement raisonnable peut dans certains établissements s'expliquer par l'importance prise par les questions de maintien dans l'emploi. Dans les établissements hospitaliers en particulier, il existe en effet une certaine confusion entre inaptitude et handicap. Un amalgame est parfois fait entre les actions pour le reclassement obligatoire pour inaptitude, c'est-à-dire le placement sur un emploi adapté aux capacités de salariés reconnus inaptes suite à un accident 
du travail ou à une maladie professionnelle et une politique d'emploi des personnes handicapées plus globale. Les employeurs, très ancrés dans une approche centrée sur l'individu, peinent à adopter une approche interactionnelle du handicap, qui intègre leurs obligations de mise en accessibilité des emplois et de compensation du handicap pour la mise à égalité des salariés (ROBIN-OLLIVIER, 2016). Plus généralement, la mise en œuvre limitée de la notion d'aménagement raisonnable peut s'expliquer par la tension entre deux conceptions de l'égalité. L'égalité des places s'oppose à l'égalité des chances (CAIlleba, Cuevas, 2009) : suivant la première, chacun bénéficie des mêmes avantages et subit les mêmes inconvénients ; suivant la seconde, l'égalité est conçue comme un objectif à atteindre, où les différences sont compensées afin que chacun ait les mêmes opportunités.

Dans le contexte réglementaire, législatif et politique que nous venons de dépeindre, diverses tensions et zones d'incertitude constituent autant de marges de manœuvre pour les acteurs concernés. L'étude des pratiques de sept employeurs publics montre qu'ils n'ont pas manqué de s'en saisir.

\section{Des réponses variées à l'obligation d'emploi par les employeurs publics}

Depuis la loi de 2005 et le renforcement de l'obligation d'emploi, les employeurs publics se sont saisis de cette question. Le taux d'emploi de bénéficiaires a ainsi fortement augmenté, c'est-à-dire que la part des bénéficiaires dans l'effectif global des salariés publics s'est accrue. Mais derrière cette tendance moyenne et largement partagée se dessinent différentes pratiques. Les employeurs font preuve d'usages sélectifs des instruments mis à leur disposition (DoYTCHEVA, 2009). De la même manière que pour l'incitation à conclure des accords ou des plans d'action en faveur de l'emploi des seniors de 2008, au-delà des effets quantitatifs immédiatement identifiables (augmentation du nombre d'accords conclus et du taux d'emploi), des effets différenciés sur l'évolution des pratiques peuvent être observés (FARVAQUE, 2011 ; CASER, JOLIVET, 2014).

\section{Arbitrage entre stratégie catégorielle et non-discrimination}

Le poids de la contribution financière, nous l'avons vu, est souvent la première motivation pour agir pour les directions des ressources humaines. De ce fait, les employeurs peuvent se focaliser sur l'obtention de résultats visibles et donc sur l'augmentation du taux d'emploi. Tous ont l'obligation de déclarer annuellement le nombre de BOE qu'ils emploient. Pour certains, cette action est centrale et concentre

5. Art. 71 à 76 de la Loi $n^{\circ} 86-33$ du 9 janvier 1986 portant dispositions statutaires relatives à la fonction publique hospitalière. 
l'essentiel des efforts fournis au nom de la politique d'emploi des personnes handicapées. Étant donné les enjeux soulevés par le fait de se déclarer travailleur handicapé, beaucoup d'agents hésitent et le recensement peut donc effectivement devenir une tâche astreignante. Or, elle peut se faire indépendamment de toute réflexion au niveau organisationnel :

« Ça a progressé parce que c'est vrai qu'on essaie de les motiver aussi à se déclarer [...]. Petit à petit, comme ça, on arrive à convaincre les gens, surtout quand on leur parle, justement, départ en retraite, éventuellement, avantage impôts, aussi, hein ! »

(Médecin de la fonction publique d'État, septembre 2015)

Parmi les employeurs enquêtés, ceux visant essentiellement l'atteinte du quota de $6 \%$ par le biais du recensement et de la déclaration des BOE concentrent généralement leurs efforts sur la question du maintien dans l'emploi, et les BOE en poste y sont donc en majorité des personnes dont le handicap a été reconnu au cours de leur exercice professionnel. C'est en particulier le cas dans les centres hospitaliers où, du fait de conditions de travail cumulant les facteurs de risque, un certain nombre d'agents développent une incapacité motrice au cours de leur emploi ; les personnes en situation de maintien dans l'emploi, de reconversion professionnelle ou de reclassement peuvent alors parfois suffire pour atteindre le quota. Comme l'ont déjà mis en avant D. THIVET et E. FiLlion (2017), l'importance numérique de ces cas s'avère être un frein à une réflexion plus axée sur les facteurs environnementaux du handicap.

Cependant, chez certains employeurs enquêtés se dessine une réflexion globale sur les situations de handicap, qui intègre l'environnement de travail de manière à permettre une égalité réelle entre personnes valides et handicapées. Peu à peu, les $\mathrm{DRH}$, les référents handicap, l'encadrement, les services sociaux et les médecins se mobilisent autour de cette question et la construisent en enjeu collectif. Les travailleurs handicapés restent en marge de ce processus bien qu'ils en bénéficient. Si le recensement et la déclaration des BOE restent incontournables, des actions pour la mise en accessibilité des locaux, pour l'adaptation des postes individuels et plus généralement pour l'instauration d'un environnement inclusif se mettent ainsi en place. Cette évolution est perceptible dans le profil des BOE que l'on rencontre dans ces structures employeuses : quand les acteurs adoptent une conception interactionnelle du handicap, les recrutements de personnes déclarant un handicap au moment de l'embauche se font plus nombreux, voire font l'objet d'actions spécifiques de recrutement. L'établissement culturel a par exemple signé une convention en 2015 avec le Greta des Métiers du tertiaire et Pôle emploi, convention dont l'objectif est de faciliter l'embauche de chômeurs de longue durée reconnus handicapés, et ainsi recruter plusieurs personnes de manière pérenne. De même que dans la collectivité territoriale 1 , ses responsables ont revu leurs procédures de recrutement et se sont fixé des objectifs sur ce plan.

Un continuum apparait de fait entre les stratégies focalisées sur le simple recensement des BOE et celles ayant pour objectif de créer un environnement de travail réellement inclusif, entre les approches resserrées sur le quota et celles intégrant la 
notion d'aménagement raisonnable. Atteindre le quota de $6 \%$ et mettre en place des aménagements raisonnables ne vont pas toujours de pair, certains employeurs se limitant à une logique quantitative, qu'accompagne parfois un traitement catégoriel spécifique des personnes jugées vulnérables. Situation a priori plus surprenante dans ce schéma, certaines organisations se mobilisent autour de la production d'égalité, mais ne comptent pas pour autant un taux élevé de BOE : certains employeurs, tel que l'établissement culturel, ont par exemple développé d'autres indicateurs pour mesurer l'efficacité de leur politique, comme la possibilité d'intégrer des personnes présentant des troubles psychiques, perçus comme le handicap le plus complexe à appréhender.

\section{Des réseaux d'acteurs variablement intégrés}

Les stratégies des employeurs publics reflètent également la difficulté de considérer le handicap comme un enjeu transversal aux organisations. En fonction de la perception de l'enjeu que représente l'emploi des personnes handicapées, ces stratégies mobilisent les acteurs à des degrés divers. Chaque catégorie de personnel présente des intérêts, des représentations et des ressources plus ou moins propices à en faire des acteurs de cette cause et à les doter d'une capacité d'action concernant l'emploi des personnes handicapées ; d'une organisation à l'autre, des réseaux d'acteurs diversement intégrés se constituent autour de cette question (HASSENTEUFEL, 2008).

Deux types d'acteurs semblent jouer un rôle majeur : les directions des ressources humaines (DRH) et les médecins du travail. L'implication des DRH sur cette question s'est particulièrement fait sentir à partir de la loi de 2005 et de la mise en place d'une contribution pour la fonction publique. En raison de leur pouvoir sur les mouvements de personnel, elles peuvent décider de la mise en place ou non d'une stratégie pour l'emploi des personnes handicapées. Elles ont un intérêt particulier à en développer une, car cela renforce leur pouvoir au sein de l'organisation :

« Ça mélange tous les avantages pour une DRH. Vraiment, on met en œuvre des actions au bénéfice d'agents de l'établissement, donc de l'accompagnement, du financement. On accompagne l'encadrement qui est très démuni et très en difficulté, parfois, sur cette thématique. C'est une politique mesurable, donc, on suit notre nombre de BOE, les actions qu'on a financées, notre taux d'utilisation de notre convention, notre taux d'emploi, l'évolution de notre contribution. Il n'y a pas beaucoup de politiques RH dont on peut mesurer les résultats comme ça et communiquer dessus et, en plus, montrer au reste de la communauté que c'est bénéfique pour les agents, mais aussi pour l'institution. »

(DRH de la fonction publique hospitalière, septembre 2015)

L'intérêt à s'impliquer est plus fort pour les directions des ressources humaines qui disposent déjà de ressources au sein de leur organisation, ont mis en place d'autres actions autour de la prévention des risques, de la montée en compétences, des départs en retraite ou autres, et ont donc un pouvoir sur la gestion des personnels. Celles dont le pouvoir est faible sont limitées dans leur implication. 
Quant aux médecins du travail, ils ont un rôle historique sur les enjeux liés à l'emploi des personnes handicapées. Ils ont en effet la responsabilité de vérifier la compatibilité de l'état de santé de l'agent avec son poste et ses conditions de travail. Cependant, le temps dont ils disposent et leur emplacement au sein ou à l'extérieur de la structure peuvent limiter leur implication. De plus, leur positionnement est souvent complexe. S'ils jouissent d'une place prépondérante en tant que responsables de l'adaptation des postes, des techniques et des rythmes de travail en fonction de la santé physique et mentale de l'agent, ils doivent de plus en plus collaborer avec d'autres catégories de professionnels : DRH, référent handicap, service social, etc. Or, ils sont tenus au secret médical et celui-ci n'est pas toujours bien compris par les autres acteurs. Des tensions existent donc au sein de certaines organisations autour du partage d'informations.

À cet égard, la position des référents handicap entre DRH et médecin du travail est révélatrice. Une de leurs fonctions est d'assurer une médiation, de coordonner les interactions entre les acteurs. Généralement rattachés aux ressources humaines, ils nouent de ce fait des rapports particuliers avec ce service et sa direction. Ce rattachement leur offre des informations et une crédibilité. Cependant, dans les sept structures étudiées, ils disposent d'une autonomie importante et marquent toujours leurs distances, au cours des entretiens, vis-à-vis du service des ressources humaines et de la mauvaise réputation qui lui est parfois attachée. Leurs missions d'accompagnement des agents les amènent à obtenir des informations confidentielles et donc à partager un certain nombre de renseignements clés avec les médecins, ce qui facilite leur dialogue. Ces ressources tirées de leur position varient cependant en fonction du temps dont ils disposent et du pouvoir qui leur est effectivement concédé. À titre d'exemple, le poids du référent handicap du ministère étudié est très faible : s'il dispose des services d'une assistante, il a trop peu de temps pour obtenir des informations et agir auprès d'agents qui sont répartis sur l'ensemble du territoire national où il n'a aucun relais officiel. À l'inverse, les référents handicap ayant du temps et une position établie dans leur organisation peuvent jouer un rôle moteur dans le développement de la stratégie pour l'emploi des personnes handicapées. Le type d'actions entreprises par le référent handicap influence grandement la stratégie organisationnelle adoptée : catégorielle ou de non-discrimination.

Le rôle dévolu à l'encadrement et aux travailleurs handicapés est également significatif. Leur participation est-elle encouragée selon une vision globale de la situation de handicap, ou réduite au minimum ? On note sur ce point que si la plupart des DRH, des référents handicap et des médecins disent vouloir que les agents handicapés soient acteurs de leur situation, ces travailleurs ne sont pas pour autant des acteurs de la question de l'emploi des personnes handicapées au sens sociologique du terme. Ils constituent surtout des ressources pour les DRH, les médecins et les référents handicap, dont le pouvoir dépend en grande partie de leur capacité à obtenir des informations de leur part ainsi que des encadrants et à pouvoir légitimement parler en leur nom. 
Selon leur degré d'implication, les différents acteurs sont mis en relation par divers types d'interactions autour de la question de l'emploi des personnes handicapées, allant d'interactions rares et/ou conflictuelles à des interactions nombreuses constituant un réseau d'acteurs intégré dans une stratégie organisationnelle. Les deux centres hospitaliers étudiés, la collectivité territoriale 1 et l'établissement culturel ont ainsi mis en place des outils pour faciliter la coopération entre les acteurs et notamment permettre le partage d'informations. Les centres hospitaliers ont par exemple instauré des réunions mensuelles généralement appelées « commissions de maintien dans l'emploi », qui réunissent référents handicap, $\mathrm{DRH}$, médecin du travail et agents du service social dans le but de discuter des cas individuels et de coordonner la mise en place des aménagements de poste. À l'inverse, au sein du ministère, de l'établissement d'enseignement ou encore de la collectivité territoriale 2 , on constate une faible implication des acteurs et une méconnaissance des enjeux. Les interactions entre les acteurs y sont rares et se concentrent sur la gestion des situations d'urgence.

\section{Vers une politique de non-discrimination}

En analysant de manière croisée l'implication des acteurs et le choix d'orientation stratégique, on peut situer les pratiques des employeurs sur une échelle allant d'interactions faibles centrées sur l'atteinte du quota de travailleurs handicapés à une politique ambitieuse de non-discrimination s'appuyant sur un réseau d'acteurs intégré, en passant par une politique catégorielle active.

À une extrémité de cette échelle, comme exposé ci-dessus, le ministère, l'établissement d'enseignement supérieur et de recherche et la collectivité territoriale 2 présentent des actions essentiellement liées à l'atteinte des $6 \%$ de BOE et se caractérisent par un faible nombre d'acteurs mobilisés sur les questions d'emploi des personnes handicapées. Au sein de ces établissements, les stratégies organisationnelles en matière de handicap sont peu structurées, faiblement formalisées ; la logique de non-discrimination n'y a que peu de place et peu de ressources lui sont consacrées. Les rares documents faisant état de leur stratégie en la matière sont succincts. Les agents chargés de missions clairement en lien avec le handicap sont rares, et aucun espace de coopération n'est consacré à ces questions : les interactions sont réduites au strict minimum. La faiblesse des ressources des référents handicap est notoire : ils sont rarement identifiés en tant que tels par les personnels, le temps dont ils disposent sur ces missions est jugé insuffisant, de telle sorte qu'ils gèrent essentiellement le recensement des BOE et les urgences.

Dans un entre-deux, les deux centres hospitaliers présentent une politique catégorielle marquée, appuyée sur un réseau d'acteurs très intégré. DRH, médecin du travail, référent handicap, service social et directeurs de pôle sont fortement mobilisés sur ces enjeux. Leurs interactions sont formalisées dans un document faisant état de la stratégie pour favoriser l'emploi de personnes handicapées. La participation du personnel d'encadrement et des agents bénéficiaires de l'obligation d'emploi est encouragée. 
Cependant, la stratégie organisationnelle de ces établissements est largement centrée sur les actions visant le maintien dans l'emploi d'agents ayant acquis leur handicap au cours de leur exercice. La réflexion sur la création de conditions de travail adaptées à tous est encore embryonnaire.

Enfin, l'établissement culturel et la collectivité territoriale 1 dépassent peu à peu la logique de quota pour développer une stratégie réellement orientée vers une politique de non-discrimination. Cette stratégie s'appuie notamment sur une culture de la santé et du bien-être au travail, c'est-à-dire sur une réflexion historique concernant les conditions de travail. La stratégie adoptée par ces établissements traduit une approche du handicap qui se veut globale et mettant en avant la nécessaire implication de tous pour le développement d'un environnement de travail inclusif. Volontaristes, les responsables s'organisent pour répondre à ce qui apparaît comme un « défi organisationnel » et non un simple enjeu individuel attaché à la personne en situation de handicap (LE Roy-Hatala, 2009). Une telle structuration du réseau d'acteurs et une telle approche du handicap sont notamment permises par un important budget dédié à la politique du handicap.

\section{Quels facteurs pour expliquer cette variété de situations ?}

Si les DRH, les médecins du travail et les référents handicap peuvent choisir de développer les stratégies qu'ils souhaitent pour favoriser l'emploi des personnes handicapées, la mise en œuvre d'une logique de non-discrimination et la mobilisation d'un réseau d'acteurs dépend tout de même d'un certain nombre d'éléments contextuels (tableau 2), en particulier : les enjeux économiques et financiers; les secteurs d'activité et les cultures professionnelles dont ils sont porteurs ; l'importance des normes bureaucratiques régissant l'emploi dans la fonction publique et la conception de l'égalité qu'elles véhiculent ; enfin, la taille des organisations.

\section{Les contraintes économiques et financières, un critère primordial}

Comme l'ont montré Jeb BARNES et Thomas F. Burke (2006 ; 2012), la crainte de sanctions et les ressources organisationnelles sont cruciales dans la mise en place des réponses à la législation en matière d'emploi des personnes handicapées. Cependant, dans notre étude, les exigences économiques et financières semblent prédominer. $\mathrm{La}$ mise en place d'actions et la mobilisation des acteurs paraissent largement dépendre de la perception de l'enjeu financier associé à l'effort envisagé ou conduit en faveur de l'emploi des personnes handicapées. Les acteurs se montrent d'autant plus investis qu'ils y voient un moyen intéressant de réunir des fonds grâce aux aides du FIPHFP, ou de réduire des dépenses en baissant la contribution versée en cas de non-respect du taux de $6 \%$. 
TABLEAU 2 - Caractéristiques clés des employeurs étudiés

\begin{tabular}{|c|c|c|c|c|}
\hline & $\begin{array}{c}\text { Enjeux } \\
\text { économiques } \\
\text { et financiers }\end{array}$ & $\begin{array}{l}\text { Taille (nombre } \\
\text { d'agents) }\end{array}$ & $\begin{array}{c}\text { Formes } \\
\text { d'organisation }\end{array}$ & Secteur d'activité \\
\hline Centre hospitalier Alpha & $\begin{array}{l}\text { Fortes contraintes } \\
\text { budgétaires }\end{array}$ & $\begin{array}{l}\text { Entre } 1000 \text { et } \\
5000\end{array}$ & $\begin{array}{c}\text { Bureaucratisation } \\
\text { moyenne } \\
\text { Travail d'équipe }\end{array}$ & Soins aigus \\
\hline Centre hospitalier Beta & $\begin{array}{l}\text { Fortes contraintes } \\
\text { budgétaires }\end{array}$ & $\begin{array}{l}\text { Entre } 1000 \text { et } \\
5000\end{array}$ & $\begin{array}{c}\text { Bureaucratisation } \\
\text { moyenne } \\
\text { Travail d'équipe }\end{array}$ & $\begin{array}{l}\text { Prise en charge } \\
\text { psychiatrique }\end{array}$ \\
\hline Collectivité territoriale 1 & $\begin{array}{l}\text { Convention avec } \\
\text { montant d'aides } \\
\text { important }\end{array}$ & $\begin{array}{l}\text { Entre } 1000 \text { et } \\
5000\end{array}$ & $\begin{array}{l}\text { Faible } \\
\text { bureaucratisation } \\
\text { Travail individuel } \\
\text { et travail d'équipe }\end{array}$ & $\begin{array}{l}\text { Administration } \\
\text { locale }\end{array}$ \\
\hline Collectivité territoriale 2 & $\begin{array}{c}\text { Convention signée } \\
\text { par le centre de } \\
\text { gestion }\end{array}$ & Moins de 250 & $\begin{array}{c}\text { Faible } \\
\text { bureaucratisation } \\
\text { Travail d'équipe }\end{array}$ & $\begin{array}{l}\text { Administration } \\
\text { locale }\end{array}$ \\
\hline Ministère Y & $\begin{array}{c}\text { Exempté de } \\
\text { contribution et } \\
\text { montant d'aides } \\
\text { par agent faible }\end{array}$ & $\begin{array}{l}\text { Plus de } \\
20000\end{array}$ & $\begin{array}{c}\text { Forte } \\
\text { bureaucratisation } \\
\text { Travail individuel }\end{array}$ & $\begin{array}{l}\text { Administration } \\
\text { centrale }\end{array}$ \\
\hline $\begin{array}{l}\text { Établissement d'enseignement } \\
\text { supérieur et de recherche }\end{array}$ & Pas de convention & Entre 250 et 1000 & $\begin{array}{c}\text { Forte } \\
\text { bureaucratisation } \\
\text { Travail individuel } \\
\text { majoritaire }\end{array}$ & $\begin{array}{l}\text { Enseignement } \\
\text { supérieur et } \\
\text { recherche }\end{array}$ \\
\hline Établissement culturel Chêne & $\begin{array}{c}\text { Convention avec } \\
\text { montant d'aides } \\
\text { important }\end{array}$ & Entre 250 et 1000 & $\begin{array}{c}\text { Faible } \\
\text { bureaucratisation } \\
\text { Travail d'équipe } \\
\text { majoritaire }\end{array}$ & Service culturel \\
\hline
\end{tabular}

Source : données fournies par les établissements en 2015 ; enquête des auteures.

Ainsi, la perception de la contribution versée au FIPHFP est déterminante et dépend largement du contexte économique et financier de l'établissement. Partout, les budgets alloués sont en baisse. La logique de rentabilité propre au secteur privé s'impose en effet peu à peu au secteur public (ALBER, 2013). Mais dans certains établissements, ce resserrement budgétaire est ressenti de manière accentuée, de telle sorte que la nécessité de réduire la contribution au FIPHFP en augmentant le taux d'emploi de bénéficiaires dans l'effectif global est jugée primordiale. L'hôpital Alpha est ainsi particulièrement impacté par le changement de modèle économique introduit par la tarification à l' activité ${ }^{6}$. Les contraintes budgétaires et les réorganisations imposées par ces réformes ont été rappelées à plusieurs reprises par les enquêtés. Elles ont des effets

6. La tarification à l'activité est le mode de financement introduit par le plan Hôpital 2007 pour les activités de médecine, de chirurgie et d'obstétrique. Les ressources allouées aux établissements sont calculées en fonction du nombre d'actes ; le prix de chaque activité étant fixé annuellement par le ministère chargé de la Santé. Auparavant, les établissements publics de santé ainsi que les établissements privés participant au système public de santé percevaient une dotation globale calculée en fonction du nombre de journées. 
ambivalents. L'impératif de réduire la contribution pousse certes à agir. Cependant, les contraintes budgétaires peuvent constituer un obstacle à l'emploi de personnes handicapées. En effet, quand la focalisation sur l'objectif d'efficience est forte, les conditions d'exercice effectives de l'activité sont rarement questionnées et la gestion des ressources humaines est centrée sur la recherche de travailleurs aux profils-types répondant parfaitement aux exigences de rentabilité.

De l'autre côté, le montant des aides obtenues par le FIPHFP conditionne la capacité à mobiliser les acteurs et à en faire un réseau intégré. Les deux plus petits employeurs étudiés que sont la collectivité 2 et l'établissement d'enseignement n'ont pas la possibilité de signer une convention avec le FIPHFP et les montants d'aides qu'ils perçoivent via cette plateforme sont relativement faibles. Le ministère, bien qu'ayant pu signer une convention avec le FIPHFP, est également contraint par le montant des aides perçues : si dans l'absolu, celui-ci est élevé, rapporté au nombre d'agents, il est trois fois inférieur au montant des aides par agent perçues par le centre hospitalier Alpha et dix-sept fois inférieur à celui obtenu par l'établissement culturel. Or, ces faibles montants coïncident avec une faible mobilisation. Si cette dernière peut expliquer la faiblesse des aides, on peut aussi penser qu'à l'inverse, le faible montant d'aides freine la mobilisation des acteurs et l'élargissement de la réflexion à une logique de non-discrimination.

Le montant des aides semble également influencer la manière d'appréhender le handicap et le paradigme guidant la politique organisationnelle. Les ressources financières sont en effet essentielles pour permettre de créer un environnement inclusif grâce à la mise aux normes du cadre bâti et à l'adaptation des postes de travail aux besoins particuliers des agents. La collectivité territoriale 1 et l'établissement culturel, qui se sont engagés dans ce type d'actions, sont les deux établissements ayant les montants d'aide par agent les plus élevés.

\section{Des métiers intrinsèquement moins favorables que d'autres ?}

Si les aspects économiques et financiers nous semblent importants, le premier critère mis en avant par les enquêtés est celui des métiers :

« On protège les malades, il faut qu'il y ait des soignants en bon état, en bien-être psychique. Comment on peut, sinon, soigner... Et je trouve que c'est essentiel, vraiment !»

(Mariette, cadre de santé de la fonction publique hospitalière, septembre 2015)

« Mais ce n'est pas Madeleine en tant que personne handicapée, le problème n'était pas là, c'était qu'il y avait un contexte qui faisait que c'était compliqué. Parfois, on a des exigences de poste qui font que ça ne correspond pas forcément avec le fait d'avoir un handicap. Une personne comme elle, par exemple, qui a besoin dans son poste de se déplacer beaucoup et qui a ce handicap-là, c'est un drôle de souci. »

(Joëlle, responsable ressources humaines, fonction publique d'État, novembre 2015) 
Interrogée sur le faible taux de travailleurs handicapés : «On recrute beaucoup de catégories A, et des profs. C'est vrai que jusqu'à présent, on n'a jamais eu de candidature de prof, si, une ! Une ! Mais qu' on ne l'a pas retenue parce que, justement, il était dans un jury normal et qu'il y avait un autre candidat qui répondait beaucoup mieux au profil. »

(Nicole, DRH de l'établissement d'enseignement supérieur et de recherche, avril 2015)

Nos résultats rejoignent ceux de l'étude de Christophe EVERAERE (2010) sur les magasins de bricolage, qui montrent que les métiers ayant une double composante physique et relationnelle sont difficilement associés à l'emploi de personnes handicapées. Les représentations tant des métiers que du handicap freinent ainsi l'emploi des personnes handicapées sur les métiers cumulant les facteurs de pénibilité et impliquant une relation proche avec les usagers, comme ceux de l'enseignement ou du soin. Ainsi, bien que les travailleurs handicapés rencontrés se répartissent sur différents types d'activité (entretien des espaces verts, travail administratif, surveillance, etc.), nous n'avons rencontré aucun soignant ou chercheur et une seule enseignante qui soient salariés au titre de BOE. Les métiers à responsabilités, notamment managériales, sont également jugés peu compatibles avec ce type de travailleurs. En effet, si certains agents handicapés que nous avons approchés étaient très autonomes, voire experts dans leur domaine, aucun n'avait de fonction d'encadrement. De façon générale, les personnes handicapées sont surreprésentées dans les postes peu qualifiés : parmi les personnes rencontrées en tant que BOE, huit étaient agents de catégorie $\mathrm{A}$, cinq de catégorie B et dix-neuf de catégorie C. Ces données rejoignent celles des enquêtes nationales : l'enquête Handicap santé ménages de 2008 montre que si $46 \%$ des agents de la fonction publique non-handicapés sont de catégorie C, c'est le cas de $70 \%$ des agents handicapés ; le module ad hoc de l'enquête Emploi de 2011 dresse le même constat et montre que si $57 \%$ des agents non-handicapés sont de catégorie C, c'est le cas de $77 \%$ des agents handicapés (VALDÈs, 2016).

\section{Taille et bureaucratisation : des contraintes spécifiques à la fonction publique}

Comme l'a montré Nathalie BAYLE (2000), la structure de l'organisation est un facteur important pour l'inclusion des travailleurs handicapés par les employeurs : un fort degré de formalisation peut être protecteur pour les salariés et, dans le même temps, figer les relations entre les individus et ne permettre l'intégration des personnes qu'à condition que leur état de santé et leurs capacités leur permettent de respecter les normes sociales, tandis qu'une faible formalisation peut s'avérer moins protectrice et en même temps permettre une plus forte influence des décideurs et laisser davantage de place aux initiatives pour l'emploi des personnes handicapées. Or, la fonction publique se caractérise par un haut degré de formalisation, c'est-à-dire par des organigrammes comportant de nombreux échelons, des processus décisionnels centralisés, une forte planification des activités, etc. Les aspects liés à la gestion des ressources humaines, du recrutement jusqu'à l'évolution de carrière, sont fortement cadrés par les statuts de 
la fonction publique. Certains employeurs publics, comme les hôpitaux, sont encore plus marqués par cette formalisation : les procédures y sont d'autant plus prégnantes et les normes d'autant plus fortes qu'elles sont enseignées dans des écoles spécialisées et que leur acquisition est validée par un diplôme nécessaire à l'exercice des emplois. À l'inverse, certains employeurs publics étudiés montrent un degré de formalisation moins élevé et une intégration de personnes handicapées ainsi rendue plus aisée. Par exemple, l'établissement culturel présente une organisation plus souple : le recours aux emplois contractuels y est élevé et l'importance du recrutement sur concours décroît peu à peu. Les procédures propres à la fonction publique sont ainsi peu à peu délaissées. Une telle évolution peut constituer un facteur de fragilisation de l'organisation, mais également ouvrir le champ des possibles en matière de recrutement et de management et faciliter ainsi, dans une certaine mesure, l'emploi de personnes handicapées. Le référent handicap, connu de tous et suivi dans ses démarches, use de cette souplesse pour mettre en place des actions structurelles.

Il est à noter par ailleurs que le concours est un enjeu dans les relations entre les agents. Avoir ou ne pas avoir le concours requis conditionne souvent l'appréciation mutuelle :

« On pouvait me reprocher de ne pas être rentrée [par concours] [...]. On ne passe pas partout le [concours]... On sait bien que passer un concours, c'est difficile. Du coup, je me suis sentie fière en 2012 quand j'ai eu mon admissibilité. Et puis d'ailleurs, j'ai été, en 2014, j'ai été admise. J'ai à nouveau été admissible et j'ai été... j'ai été reçue à l'oral donc, j'étais encore deux fois plus fière. »

(Agent BOE de la fonction publique d'État, mars 2015)

«Il y a toujours des agents qui ne comprennent pas pourquoi, eux, on les recrute sans concours alors qu'eux-mêmes ont dû passer des concours. »

(Assistante Mission handicap, fonction publique d'État, septembre 2015)

Cette question du concours pose plus généralement la question de la définition de l'égalité intrinsèquement portée par la fonction publique. Le concours, ainsi que les grilles indiciaires, sont en effet présentés comme des gages d'égalité ; mais il s'agit d'une égalité de principe alors que l'objectif d'emploi des personnes handicapées appellerait à prendre en compte les différences en menant des actions de compensation pour permettre une égalité réelle. Des adaptations sont proposées aux personnes reconnues handicapées pour les aider à passer les concours. Malgré cela, les marges de manœuvre restent minimes et ne permettent que difficilement la prise en compte de la spécificité des situations individuelles. Une agente aveugle qui avait tenté de passer des concours de la fonction publique territoriale nous a par exemple expliqué l'incompatibilité de l'épreuve de la note de synthèse avec son handicap : la synthèse vocale qu'elle utilise ne lui offre par exemple pas une navigation souple entre les documents alors que cela est décisif dans une telle épreuve.

Enfin, la taille de l'employeur en termes d'effectif apparait comme un paramètre essentiel. Une taille plus importante amène généralement à la constitution d'un service 
des ressources humaines plus important voire à la création d'un poste dédié aux questions de handicap et de reclassement. En somme, les plus grandes organisations ont plus de marges de manœuvre, notamment dans leur offre de reclassement :

« On est une grosse collectivité. Donc, on a aussi des possibilités que n'ont pas les petites communes, par exemple. C'est compliqué quand on est dix d'avoir, je ne sais pas, moi, la personne qui s'occupe des rues, elle ne peut plus travailler parce qu'elle a mal au dos machin, on va avoir du mal à la repositionner sur un poste administratif, parce qu'ils en ont déjà eux, ils en ont deux ! Ils n'ont pas besoin d'être trois! Donc, on a aussi des marges de manœuvre parce qu'on est très nombreux. Et qu'on a beaucoup de métiers : on a à peu près 287 métiers, on est plus de 5000 agents, donc...»

(Référente handicap, fonction publique territoriale, décembre 2014)

« Ben, ici, l'avantage, c'est qu'on est un établissement moyen en termes d'effectifs [...]. On a une bonne taille c'est-à-dire qu'à la fois, les gens arrivent à se connaître mais en même temps, on n'est pas les uns sur les autres, quoi. C'est vrai que les toutes petites équipes, ce n'est pas facile à gérer non plus, hein. »

(Directeur de l'accueil et de la sécurité, fonction publique d'État, novembre 2015)

À l'extrême, une trop grande taille peut cependant devenir un frein. La multiplication des échelons hiérarchiques peut freiner les prises de décision et leur application. Or, les effectifs des employeurs publics sont particulièrement importants en comparaison de ceux des entreprises du secteur privé. Les ordres de grandeur sont très différents : on dit d'un hôpital qui compte 2500 agents qu'il est de taille moyenne, d'un établissement public qui compte quelques 400 agents qu'il est petit alors qu'une entreprise est considérée comme de taille intermédiaire au-delà de 250 agents et comme grande au-dessus de 5000 salariés $^{7}$. Or, l'exemple du ministère nous montre qu'une trop grande taille, associée à une répartition du personnel sur l'ensemble du territoire, est un frein à la mobilisation des personnes ressources et à la constitution d'un réseau d'acteurs intégré pour permettre l'emploi des personnes handicapées.

Le mode de gestion des ressources humaines de la fonction publique présente ainsi des effets ambivalents : s'il s'avère protecteur dans certains cas, il peut également avoir des effets indésirables. La forte formalisation des organisations du travail et la très grande taille des établissements semblent freiner les initiatives en matière d'emploi des personnes handicapées. De même, le mode de recrutement par concours paraît inadapté pour un certain nombre de personnes handicapées. Le mode de recrutement par voie contractuelle en raison du handicap n'est pas non plus sans poser question, ce qui rejoint la situation observée dans le secteur privé. La fonction publique rencontre quoi qu'il en soit aujourd'hui un certain nombre de difficultés pour parvenir à une égalité des chances réelle. Comme l'ont montré A. CoRnet et M. El ABOuBi (2012), elle présente en ce sens une situation de « discrimination systémique ». Nous formulons cependant

7. Décret $n^{\circ} 2008-1354$ du 18 décembre 2008 relatif aux critères permettant de déterminer la catégorie d'appartenance d'une entreprise pour les besoins de l'analyse statistique et économique. 
l'hypothèse que cela ne constitue pas une spécificité de la fonction publique. Il est en effet fortement probable qu' une discrimination semblable existe dans la majorité des structures, qu'elles soient publiques ou privées, comme le suggèrent les différents travaux conduits sur le secteur privé évoqués en introduction.

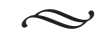

Si l'emploi des personnes handicapées a progressé dans la fonction publique depuis l'adoption de la loi de 2005, ce constat doit cependant être nuancé. Nos travaux montrent que nous sommes face à une réalité complexe. Alors que le droit international, par la voie de la Convention pour les droits des personnes handicapées de 2006 ou encore de la directive européenne en faveur de l'égalité de traitement en matière d'emploi et de travail de 2000, invite à l'adoption d'une logique de production d'égalité et de non-discrimination, les employeurs étudiés sont encore très ancrés dans une logique catégorielle basée sur le quota de travailleurs handicapés. De plus, la mobilisation des acteurs reste très partielle : si chaque structure étudiée comprend au moins une personne en charge des questions liées à l'emploi des personnes handicapées, très peu disposent d'un réseau d'acteurs intégré mobilisé sur ces enjeux.

La question de l'évolution et du devenir des politiques du handicap en matière d'emploi se pose avec d'autant plus d'acuité, notamment dans la fonction publique, que les taux d'emploi augmentent et tendent vers les $6 \%$ requis. Or, plus les employeurs se rapprochent du taux à atteindre, moins ils versent de contributions. Les ressources financières du FIPHFP ont donc drastiquement baissé et se trouvent dès à présent fortement limitées. La pérennité économique même de ces politiques est donc en jeu. Le développement d'une politique de non-discrimination et le renforcement de ses instruments, notamment de la notion d'aménagement raisonnable, constituent une réponse potentielle à cette situation. Dans les faits, la prégnance du quota dans les pratiques apparaît actuellement comme un frein à cette évolution. Par ailleurs, si distinguer logique de non-discrimination et logique catégorielle aide à analyser les tendances, l'analyse des politiques publiques du handicap nous enseigne que dans la pratique il n'existe pas d'opposition radicale entre les deux, qui bien souvent se combinent (BAUDOT et al. 2013). En l'occurrence, la mobilisation d'acteurs autour de l'objectif des $6 \%$ peut être une base pour une réflexion plus globale sur l'instauration de conditions de travail adaptées à tous. Il serait intéressant d'étudier plus en détail les facteurs qui amènent les acteurs à élargir leur réflexion et leurs pratiques.

Le développement d'une logique de non-discrimination universelle est complexe dans le contexte français. Il ne saurait s'effectuer en matière d'emploi sans une réflexion sur la place et le sens du travail. Le défi est particulièrement relevé dans la fonction publique, où la prise en compte des besoins spécifiques des travailleurs handicapés autour de la notion d'aménagement raisonnable vient questionner la vision de l'égalité qui y est aujourd'hui portée. 


\section{BIBLIOGRAPHIE}

Alber A. (2013), « Management et nouvelle gestion publique : limites et paradoxes de l'imitation du privé », La Nouvelle Revue du travail, ${ }^{\circ} 2$; en ligne : http://nrt.revues.org/934.

BAREL Y., FrÉmEAuX S. (2013), «Le management du travail, condition de réussite du management de la diversité », Management \& Avenir, $n^{0}$ 66, pp. 85-102.

Barhoumi M., Chabanon L. (2015), «Emploi et chômage des personnes handicapées », Synthèse Stat', novembre, $\mathrm{n}^{\circ} 17$.

Barnay T., Duguet E., Le Clainche C., Narcy M., Videau Y. (2015), “The Impact of Handicap on Occupations: A Comparison Between Public and Private Sectors", Annals of Economics and Statistics, $\mathrm{n}^{\circ} 119-120$, pp. 39-64.

Barnes J., Burke T. F. (2006), "The Diffusion of Rights: From Law on the Books to Organizational Rights Practices", Law \& Society Review, vol. 40, n³, pp. 493-524.

BARnES J., Burke T. F. (2012), "Making Way: Legal Mobilization, Organizational Response and Wheelchair Access", Law \& Society Review, vol. 46, n 1, pp. 167-198.

BARril C., MetzGer, J.-L. (2004), «L'insertion professionnelle des travailleurs aveugles et sourds : les paradoxes du changement technico-organisationnel », Revue française des affaires sociales, $\mathrm{n}^{\mathrm{o}} 3$, pp. 63-86.

BAUdOT P.-Y. (2016), « Le handicap comme catégorie administrative. Instrumentation de l'action publique et délimitation d'une population », Revue française des affaires sociales, $\mathrm{n}^{\circ} 4$, pp. 63-87.

Baudot P.-Y., Borelle C., Revillard A. (2013), « Le voyage des droits. Introduction à la traduction de Rights or quotas ? », Terrains et travaux, n 23, pp. 113-125.

BAYLE N. (2000), Entre le penser et le faire. La production des comportements d'inclusion des travailleurs handicapés par les employeurs, Lille, ANRT.

Bertrand L., Caradec V., Eidelimann J.-S. (2014), « Devenir travailleur handicapé, enjeux individuels, frontières institutionnelles », Sociologie, vol. 5, n ${ }^{\circ}$ 2, pp. 121-138

Cailleba P., Cuevas, F. (2009), « Retour d'expériences sur le management de la diversité dans 250 entreprises françaises », Management \& Avenir, $\mathrm{n}^{\circ}$ 28, pp. 366-379.

CAlvès G. (2016), La Discrimination positive, Paris, Presses universitaires de France, coll. «Que sais-je?».

CAser F., Jolivet A. (2014), «L'incitation à négocier en faveur de l'emploi des seniors. Un instrument efficace ? », La Revue de l'Ires, ${ }^{\circ}$ 80, pp. 27-48.

Cornet A., El Abboubi M. (2012), «Gérer la diversité dans le secteur public : pratiques et conditions de succès », Gestion, vol. 37, n 4, pp. 57-66.

DALle-NAzÉBi S. (2013), «Récits d'une violence ordinaire. Les sourds au travail », Développement humain, handicap et changement social, vol. 18, nº 2, pp. 43-58.

DAlle-Nazébi S., Kerbourc'h S. (2013), « L'invisibilité du "travail en plus” de salariés sourds », Terrains et travaux, vol. 2, n ${ }^{\circ} 23$, pp. 159-177. 
DÉFENSEUR DES DroITs (2017), Rapport annuel d'activité 2016 ; en ligne : https://www.defenseurdesdroits.fr/fr/rapports-annuels-dactivite/2017/02/rapport-annuel-dactivite-2016.

DGAFP (2015), Fonction publique, Chiffres-clés 2015, Paris, Ministère de la Décentralisation et de la Fonction publique, coll. « Faits et chiffres ».

Doytcheva M. (2009), « Réinterprétations et usages sélectifs de la diversité dans les politiques des entreprises », Raisons politiques, n $^{\circ} 35$, pp. 107-124.

EVERAERE C. (2010), « Insertion et maintien dans l'emploi des personnes handicapées », Revue française de gestion, vol. $3, \mathrm{n}^{\mathrm{o}} 202$, pp. 13-31.

Ewick P., Silbey S. (1998), The Common Place of Law: Stories from Everyday Life, Chicago, University of Chicago Press.

FARVAQUE N. (2011), « Le bricolage du maintien dans l'emploi des seniors : régulation publique, dialogue social et boîte à outils », La Revue de l'Ires, 69, n² 2, pp. 139-172.

GARDIEN E. (2006), « Travailleur en situation de handicap : de qui parle-t-on ? », Reliance, $\mathrm{n}^{\mathrm{o}} 1$, pp. 50-59.

Hassenteufel P. (2008), Sociologie politique : l'action publique, Paris, Armand Colin, coll. «U Sociologie».

HEYER K. (2013), « Droits ou quotas ? L'American with disabilities act (ADA) comme modèle des droits des personnes handicapées », Terrains \& travaux, vol. 2, n² 23, pp. 127-158.

Kerbourc' h S., DALle-NAZÉBI S. (2014), « Sourds au travail : la communication en entreprise au prisme de la loi sur le handicap », Connaissance de l'emploi, $\mathrm{n}^{\circ} 113$.

Lascoumes P., Le Galès P. (2010), Sociologie de l'action publique, Paris, Armand Colin , coll. « $128 »$.

Le Roy-Hatala, C. (2009), « Le maintien dans l'emploi des personnes souffrant de handicap psychique, un défi organisationnel pour l'entreprise », Revue française des affaires sociales, vol. $1, \mathrm{n}^{\circ} 1-2$, pp. 301-319.

Morin B. (2006), «L'insertion professionnelle des personnes en situation de handicap », Reliance, vol 1, $\mathrm{n}^{\circ} 19$, p. 62-66.

Point S., Charles-Fontaine C., Berthélème G. (2010), « (Re)considérer le handicap : regards croisés sur les approches en entreprise », Management \& Avenir, vol. 8, n 38, pp. 293-305.

Pouget J. (2005), « La Fonction publique : vers plus de diversité ? », Dossiers de la fonction publique, pp. 143-162.

Ravaud J.-F., MAdiot B., Ville I. (1992), “Discrimination Towards Disabled People Seeking Employment”, Social Sciences \& Medicine, vol. 35, nº 88, pp. 951-958.

RoBiN-OlLIVIER S. (2016), « La greffe de la notion d'aménagement raisonnable en droit français peut-elle réussir? » in Aménagements raisonnables et situations de handicap, quels usages d'un nouveau cadre juridique ?, Paris, EHESP, actes en ligne : http://www.ehesp.fr/wp-content/ uploads/2014/05/20160901_actes_amenagements-raisonnables-et-situations-de-handicap.pdf. 
Rowell J. (2016), « L'emploi des handicapés en Europe. Le rôle de la quantification dans l'échec d'un nouveau problème public », Genèses, n 103, pp. 96-116.

Thivet D., Fillion E. (2017), «Lutte contre les discriminations : l'exemple des personnels hospitaliers », Cahiers de la fonction publique, ${ }^{\circ} 373$, pp. 28-32.

VALDÈs B. (2016), « L'emploi des personnes handicapées dans la fonction publique en France : analyse quantitative des données d'enquêtes nationales disponibles », Revue française des affaires sociales, $\mathrm{n}^{\mathrm{o}} 4$, pp. 307-332.

Ville I., Fillion E., Ravaud J.-F. (2014), Introduction à la sociologie du handicap. Histoire, politiques et expérience, Paris, De Boeck Supérieur.

Ville I., Ravaud J.-F., Letourmy A. (2003), « Les désignations du handicap. Des incapacités déclarées à la reconnaissance administrative », Revue française des affaires sociales, $\mathrm{n}^{\circ} 1-2$, pp. 31-53.

Winance M., Ville I., Ravaud J.-F. (2007), "Disability Policies in France: Changes and Tensions between the Category-based, Universalist and Personalized Approaches", Scandinavian Journal of Disability Research, vol. 9, n 3-4, pp. 160-181. 